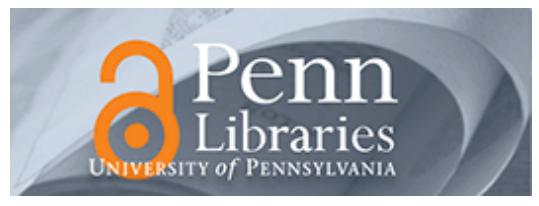

University of Pennsylvania

ScholarlyCommons

10-15-1973

\title{
Rigorous Bound on the Integrated Density of States of a Three- Dimensional Random Alloy
}

\author{
A. Brooks Harris \\ University of Pennsylvania, harris@sas.upenn.edu
}

Follow this and additional works at: https://repository.upenn.edu/physics_papers

Part of the Physics Commons

\section{Recommended Citation}

Harris, A. (1973). Rigorous Bound on the Integrated Density of States of a Three-Dimensional Random Alloy. Physical Review B, 8 (8), 3661-3664. http://dx.doi.org/10.1103/PhysRevB.8.3661

At the time of publication, author A. Brooks Harris was affiliated with Oxford University. Currently, he is a faculty member in the Department of Physics at the University of Pennsylvania.

This paper is posted at ScholarlyCommons. https://repository.upenn.edu/physics_papers/411

For more information, please contact repository@pobox.upenn.edu. 


\title{
Rigorous Bound on the Integrated Density of States of a Three-Dimensional Random Alloy
}

\author{
Abstract \\ We study the lattice model of a random alloy whose Hamiltonian is $H=-\Sigma_{r, \delta} t{ }^{\dagger}{ }_{r} a_{r+\delta}+\Sigma_{r} \varepsilon_{r} a^{\dagger}{ }_{r} a_{r}$, where $\delta$ \\ are nearest-neighbor vectors and $\varepsilon_{r}$ is a random site-diagonal energy uniformly distributed over the \\ interval $0 \leq \varepsilon_{r} \leq W$. We prove that the integrated density of states per site $N^{-1} Z(E)$ satisfies the inequality, \\ $N^{-1} Z(E) \leq C_{1} e^{-C_{2} / E}$, where $C_{1}$ and $C_{2}$ are constants. \\ Disciplines \\ Physics \\ Comments \\ At the time of publication, author A. Brooks Harris was affiliated with Oxford University. Currently, he is a \\ faculty member in the Department of Physics at the University of Pennsylvania.
}




\title{
Rigorous Bound on the Integrated Density of States of a Three-Dimensional Random Alloy*
}

\author{
A. Brooks Harris ${ }^{\dagger}$ \\ Department of Theoretical Physics, Oxford University, Oxford, England \\ (Received 13 June 1973)
}

\begin{abstract}
We study the lattice model of a random alloy whose Hamiltonian is $\mathcal{H C}=-\Sigma_{r, \delta} t a_{r}^{\dagger} a_{r+\delta}+\Sigma_{r} \epsilon_{r} a_{r}^{\dagger} a_{r}$, where $\delta$ are nearest-neighbor vectors and $\epsilon_{r}$ is a random site-diagonal energy uniformly distributed over the interval $0 \leq \boldsymbol{\epsilon}_{r} \leq \boldsymbol{W}$. We prove that the integrated density of states per site $\mathrm{N}^{-1} Z(E)$ satisfies the inequality, $N^{-1} Z(E) \leq C_{1} e^{-C_{2} / E}$, where $C_{1}$ and $C_{2}$ are constants.
\end{abstract}

\section{INTRODUCTION}

Recently, much theoretical ${ }^{1}$ and experimental ${ }^{2}$ efforts have gone into understanding the many-body properties of alloys. In this paper we study the simplest theoretical model of such an alloy, namely, one in which the hopping matrix elements are periodic, as in a pure system, but in which the site-diagonal energies are random variables. In particular, we study the case when these site-diagonal energies are uniformly distributed over the range of energy from 0 to $W$. According to the well-known argument of Lifshitz, ${ }^{3}$ one expects to realize arbitrarily large regions where the sitediagonal energies are arbitrarily close to zero. This reasoning shows that the addition of such random potentials does not change the location of the low-energy band edge. Lifshitz's argument leads to the estimate for the density of states, $\rho(E)$, at energies just above the band edge (taken to be at zero energy),

$$
\rho(E) \sim e^{-K / E^{3 / 2}},
$$

where $K$ is a constant.

In many ways the alloy problem resembles that of phase transitions. Recently, $\mathrm{Ma}^{4}$ has attempted to apply the renormalization-group arguments of Wilson ${ }^{5}$ to this problem. His result, in marked disagreement with that, [Eq. (1)] of Lifshitz, is

$$
\rho(E) \sim E^{\beta},
$$

where the critical index $\beta$ is found to be about 0.80 .

Inasmuch as Lifshitz's argument is physically so appealing, we have sought to give a rigorous proof of his estimate. Indeed, in one dimension his estimate becomes $\rho(E) \sim e^{-K^{0} / E^{1 / 2}}$ and this result has been rigorously confirmed by Eggarter. ${ }^{6}$ Although we have not been able to prove Lifshitz's result in the three-dimensional case, we have obtained rigorously the weaker result that there exists an energy $E_{0}$ such that

$$
N^{-1} Z(E) \leqslant C_{1} e^{-C_{2} / E}, \quad E<E_{0}
$$

where $C_{1}$ and $C_{2}$ are constants and $N^{-1} Z(E)$ is the in- tegrated density of states per site:

$$
N^{-1} Z(E)=\int_{0}^{E} \rho\left(E^{\prime}\right) d E^{\prime} .
$$

In view of the form of Eqs. (1) and (3) we define a critical index $\gamma$ through

$$
-\ln Z(E) \sim E^{-\gamma}, \quad E \rightarrow 0 .
$$

Then our work yields the rigorous lower bound $\gamma$ $\geq 1$ whereas Lifshitz's argument suggests that $\gamma$ $=\frac{3}{2}$. In fact, Lifshitz's value is a rigorous upper bound.

\section{PROOF OF BOUND}

The model we consider is described by the Hamiltonian

$$
\mathfrak{H}_{R}=-\sum_{r, \delta} t a_{r}^{\dagger} a_{r+\sigma}+\sum_{r} \epsilon_{r} a_{r}^{\dagger} a_{r},
$$

where $a_{r}^{\dagger}$ creates an excitation at the lattice site at $r$. For convenience we treat a simple cubic lattice in the form of a rectangular parallelepiped with $N_{x}, N_{y}$, and $N_{z}$ lattice sites on its three edges. In Eq. (6), $\delta$ is summed over the nearest-neighbor vectors of the $r$ th site. We do not introduce periodic boundary conditions, so that sites on the boundary of the system have fewer neighbors than those in the interior. Also, in Eq. (6) $\epsilon_{r}$ is a random site-diagonal energy. We assume that each $\epsilon_{r}$ is an independent random variable governed by a bounded probability distribution, i.e., one for which

$$
p(\epsilon)=0 \text { for } \epsilon<\epsilon_{L} \text { or } \epsilon>\epsilon_{G}
$$

and for which $p(\epsilon)$ is bounded for $\epsilon_{L}<\epsilon<\epsilon_{G}$. For simplicity we set

$$
\begin{array}{ll}
p(\epsilon)=W^{-1}, & 0<\epsilon<W \\
p(\epsilon)=0, & \text { otherwise. }
\end{array}
$$

From what follows it will become clear that the assumption of this special form for $p(\epsilon)$ is inessential to our arguments. We will study the density of single-particle states of $\mathcal{H}_{R}$, i.e., those for which $\sum_{r} a_{r}^{\dagger} a_{r}=1$. Accordingly, it is immaterial as to whether the $a_{r}$ 's obey Fermi or Bose statistics. 
It is convenient to shift the zero of energy, so that the band edge, when all the $\epsilon_{r}$ 's vanish, occurs at zero energy. We therefore study the Hamiltonian

$$
\mathcal{H C}\left(\left\{\epsilon_{r}\right\}\right)=\sum_{r, 6} t a_{r}^{\dagger}\left(a_{r}-a_{r+8}\right)+\sum_{r} \epsilon_{r} a_{r}^{\dagger} a_{r}
$$

Furthermore, it is convenient to study the integrated density of states $Z(E)$. For a fixed set of $\epsilon_{r}$ 's, $Z\left(E,\left\{\epsilon_{r}\right\}\right)$ is defined to be the number of single-particle states of $\mathfrak{H C}\left(\left\{\epsilon_{r}\right\}\right)$ having energy less than $E$. Ultimately, we are interested in the configurationally averaged density of states $Z(E)$, given as

$$
Z(E)=\int Z\left(E,\left\{\epsilon_{r}\right\}\right) \prod_{r}\left[p\left(\epsilon_{r}\right) d \epsilon_{r}\right] .
$$

Our proof of Eq. (3) involves repeated use of the following theorem. ${ }^{7}$

Let $H$ and $H^{\prime}$ be two $N \times N$ Hermitian matrices such that

$$
H=H^{\prime}+V \text {, }
$$

where $V$ is a non-negative $N \times N$ Hermitian matrix, and let $Z(E)$ and $Z^{\prime}(E)$ be their respective integrated density of eigenvalues. Then the set of eigenvalues of $H^{\prime}$ are lower bounds for those of $H$, or equivalently,

$$
Z(E) \leqslant Z^{\prime}(E) .
$$

Here a non-negative operator $V$ is one which can be written in bra-ket notation in the form

$$
V=\sum_{n}|n\rangle v_{n}\langle n|, v_{n} \geqslant 0
$$

We now divide the total system of lattice sites into subsystems. That is, if $\boldsymbol{\Omega}$ denotes the lattice sites of the system, we write

$$
\Omega=\sum_{i} \boldsymbol{\Omega}_{i},
$$

where $\Omega_{i}$ denotes the lattice sites of the $i$ th subsys tem, which consists of a parallelepiped of sites having $N_{x i}, N_{y i}$, and $N_{x i}$ sites on its three edges. We now show that

$$
Z_{\Omega}(E) \leqslant \sum_{i} Z_{\Omega_{i}}(E)
$$

where the subscript on the single-particle density of states indicates the system of sites to which it pertains. To prove Eq. (15) we write

$$
\mathfrak{K}_{\Omega}=\sum_{i} \mathcal{H}_{\Omega_{i}}+\sum_{i<j} V_{i j}
$$

in the notation of Eq. (15). Here the Hamiltonian of the $i$ th subsystem is

$$
\mathfrak{H}_{\Omega_{i}}=\sum_{r \in \Omega_{i}} \sum_{\delta} t a_{r}^{\dagger}\left(a_{r}-a_{r+\sigma}\right)+\sum_{r \in \Omega_{i}} \epsilon_{r} a_{r}^{\dagger} a_{r}
$$

where, as in Eq. (6), we do not impose periodic boundary conditions. The interaction between subsystems is of the form

$$
V_{i j}=\sum_{\substack{r \in \Omega^{\prime} ; \\ r^{\prime} \in \Omega^{\prime} ;}} t\left(a_{r}^{\dagger}-a_{r^{\prime}}^{\dagger}\right)\left(a_{r}-a_{r^{\prime}}\right),
$$

where the double prime indicates that only terms for which $r$ and $r^{\prime}$ are nearest neighbors are to be included. Clearly $V_{i j}$ is non-negative in the sense of Eq. (13). Hence, according to the theorem, dropping the interaction terms in Eq. (16) cannot decrease the density of states. However, in the absence of these interaction terms the density of single-particle states is simply the sum of the densities of states of the subsystems. Thus Eq. (15) is proved.

For simplicity we now introduce an inequality in terms of a discrete probability distribution for the $\epsilon_{r}$ 's. According to the theorem we can write

$$
Z\left(E,\left\{\epsilon_{r}\right\}\right) \leqslant Z\left(E,\left\{\delta_{r}\right\}\right)
$$

if $\delta_{r} \leqslant \epsilon_{r}$ for all $r$. Now we apply Eq. (19) by taking, for each $r$,

$$
\begin{aligned}
& \delta_{r}=0 \text { if } 0<\epsilon_{r}<a, \\
& \delta_{r}=a \text { if } a<\epsilon_{r}<W .
\end{aligned}
$$

Use of Eqs. (19), (20), and (8) allows us to write

$$
Z(E) \leqslant \sum_{\left\{\sigma_{r}\right\}} Z\left(E,\left\{\delta_{r}\right\}\right) \prod_{r} \hat{p}\left(\delta_{r}\right),
$$

where the $\sigma$ 's are summed over the two values 0 and $a$, and

$$
\begin{aligned}
& \hat{p}(0)=a / w \\
& \hat{p}(a)=1-\hat{p}(0) .
\end{aligned}
$$

To bound $Z(E)$ for small $E$ we choose the subsystems to be as large as possible within the restriction that

$$
A^{\prime \prime} / N_{i}>E,
$$

where $N_{i}$ is the number of sites in the $i$ th subsystem and $A^{\prime \prime}$ is the constant in Eq. (A10). Thus, we write

$$
\frac{1}{2} A^{\prime \prime} / E<N_{i}<A^{\prime \prime} / E .
$$

Furthermore, we consider energies small enough that $N_{i}$ is large enough for the bounds in Eq. (A10) to hold. With this construction it is clear that the lowest single-particle energy level of any subsys tem having one or more nonzero $\sigma$ 's has an energy greater than $E$, so that for such subsystems $Z(E$, $\left.\left\{\sigma_{r}\right\}\right)=0$. Since only subsystems having all $\sigma$ 's zero contribute to Eq. (15) we may write

$$
Z_{\Omega}(E) \leqslant \sum_{i}(a / w)^{N i} Z_{\Omega_{i}}\left(E,\left\{\sigma_{r}=0\right\}\right)
$$


We need to evaluate $Z_{\Omega_{i}}\left(E,\left\{\sigma_{r}=0\right\}\right)$, which is the density of states of the unperturbed Hamiltonian, i. e., that of Eq. (17) with all $\epsilon_{r}=0$. The singleparticle energies for this case are readily found to be

$$
E_{l, m, n}=t\left(6-2 \cos \frac{l \pi}{N_{x i}}-2 \cos \frac{m \pi}{N_{y i}}-2 \cos \frac{n \pi}{N_{z i}}\right),
$$

where $l, m$, and $n$ are integers satisfying $0 \leqslant l$ $<N_{x i} ; 0 \leqslant m<N_{y i}$; and $0 \leqslant n<N_{s i}$. Apart from $E_{000}$ the lowest single-particle energy level occurs for either $l, m$, or $n$ equal to 1 and is of order $N^{-2 / 3}$. Since $N^{-2 / 3} \gg N^{-1}$, we see that for sufficiently large $N$ we may set $Z_{\Omega_{i}}\left(E,\left\{\sigma_{r}=0\right\}\right)=1$. This evaluation, together with the inequality of Eq. (24), shows that Eq. (25) implies that there exists an $E_{0}$ such that

$N^{-1} Z_{\Omega}(E) \leqslant C_{1} e^{\left(A^{*} / E\right) \ln (a / W)}$, for $E<E_{0}$,

where $C_{1}$ is a constant. This result is equivalent to that stated in Eq. (3).

\section{DISCUSSION}

The variational principle can be used to obtain upper bounds for the energies and thence lower bounds for $Z(E)$. Use of Lifshitz's wave functions as trial functions shows that his result, [Eq. (1)], is a lower bound for $Z(E)$.

\section{ACKNOWLEDGMENTS}

The author would like to thank Dr. B. Nickel for stimulating my interest in this problem. I also appreciate the helpful comments of Dr. R. Stinchcombe and Professor R. Weinstock.

\section{APPENDIX A: MINIMUM ENERGY FOR SINGLE-DEFECT POTENTIAL}

In this Appendix we obtain rigorously a formula for the minimum single-particle energy in the presence of a single-defect potential. To do this, we use the well-known ${ }^{8}$ procedure of solving the Schrödinger eigenvalue problem by constructing the Green's function

$$
G_{E}\left(r, r^{\prime}\right)=\sum_{n} \frac{\psi_{n}^{*}(r) \psi_{n}\left(r^{\prime}\right)}{E-E_{n}},
$$

where $\psi_{n}(r)$ is the $n$th eigenfunction with energy eigenvalue $E_{n}$ when all but one of the $\sigma$ 's in Eq. (17) are zero. In particular, we take $\sigma_{r^{\prime \prime}}=a$, so that there is a site-diagonal repulsive potential at that site of strength $a$. In the absence of defect potentials, the Green's function, denoted $G_{E}^{0}\left(r, r^{\prime}\right)$, is

$$
G_{E}^{0}\left(r, r^{\prime}\right)=\sum_{n} \frac{\left[\psi_{n}^{0}(r)\right]^{*} \psi_{n}^{0}\left(r^{\prime}\right)}{\left(E-E_{n}^{0}\right)}
$$

where $\psi_{n}^{0}(r)$ and $E_{n}^{0}$ are the $n$th eigenfunction and eigenvalue, respectively, in this case.

We may express $G$ in terms of $G^{0}$ as

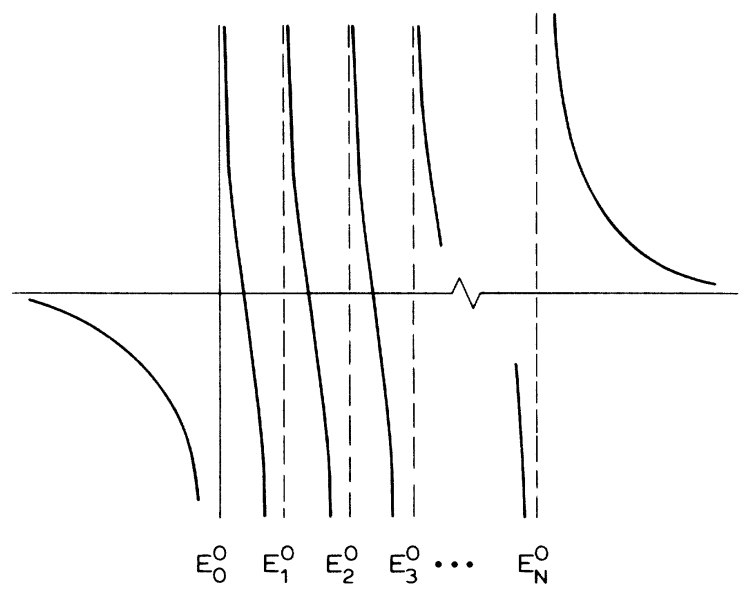

FIG. 1. Schematic behavior of $G_{E}^{0}\left(r^{\prime \prime}, r^{\prime \prime}\right)$.

$G_{E}\left(r, r^{\prime}\right)=G_{E}^{0}\left(r, r^{\prime}\right)+G_{E}^{0}\left(r, r^{\prime \prime}\right) t G_{E}^{0}\left(r^{\prime \prime}, r^{\prime}\right)$.

Here we have introduced the $t$ matrix, which satisfies

$$
t=a+a G_{E}^{0}\left(r^{\prime \prime}, r^{\prime \prime}\right) t,
$$

and thus is

$$
t=a\left[1-a G_{E}^{0}\left(r^{\prime \prime}, r^{\prime \prime}\right)\right] .
$$

Based on the fact that the poles of $G_{E}\left(r, r^{\prime}\right)$ yield the eigenvalues, an analysis of Eqs. (A3) and (A5) shows that eigenvalues occur when (i) $E=E_{n}^{0}$, in the case when $E_{n}^{0}$ is a degenerate eigenvalue of the system in the absence of defect potentials, or (ii) when $1=a G_{E}^{0}\left(r^{\prime \prime}, r^{\prime \prime}\right)$, i.e., when

$$
a^{-1}=G_{E}^{0}\left(r^{\prime \prime}, r^{\prime \prime}\right)=\sum_{n} \frac{\left|\psi_{n}^{0}\left(r^{\prime \prime}\right)\right|^{2}}{E-E_{n}^{0}} .
$$

To find the minimum single-particle eigenvalue we need only consider case (ii), i.e., Eq. (A6). Since $G_{E}^{0}\left(r^{\prime \prime}, r^{\prime \prime}\right)$ has poles at $E_{n}^{0}$, and since $d G_{E}^{0}\left(r^{\prime \prime}, r^{\prime \prime}\right) /$ $d E$ is negative for $E \neq E_{n}^{0}$, the right-hand side of Eq. (A6) has the familiar form shown in Fig. 1.

The discussion in Appendix B shows that for a three-dimensional system $E_{\infty}$, the minimum singleparticle energy for $a=\infty$, i.e., for an infinite potential at $r^{\prime \prime}$, is of order $N^{-1}$ :

$$
N E_{\infty} \sim g_{0}\left(r^{\prime \prime}\right)^{-1}, \quad N \rightarrow \infty
$$

where $g_{0}\left(r^{\prime \prime}\right)$ is defined in Eq. (B4a). For finite $a$ it is clear that $E<E_{\infty}$. A treatment similar to that leading to Eq. (B3) shows that for a three-dimensional system the minimum single-particle energy for finite $a$, denoted $E_{a}$, obeys the inequality

$$
(1-\gamma \epsilon) g_{0}\left(r^{\prime \prime}\right) \geqslant\left(N E_{a}\right)^{-1}-a^{-1} \geqslant g_{0}\left(r^{\prime \prime}\right),
$$

where $\epsilon=N E_{\infty}$ and $\gamma$ is of the order $N^{-1 / 3}$. Thus, for sufficiently large $N$ we have that $E_{a}$ is of order $N^{-1}$ : 


$$
N E_{a} \sim\left[a^{-1}+g_{0}\left(r^{\prime \prime}\right)\right]^{-1}, \quad N \rightarrow \infty .
$$

In particular, Eq. (A8) shows that for $a>0$ and for sufficiently large $N$ there exists a nonzero $A^{\prime \prime}$ such that

$$
E_{a} \geqslant A^{\prime \prime} / N \text {. }
$$

\section{APPENDIX B: PROOF THAT $E_{\infty}$ IS OF ORDER $N^{-1}$}

In this Appendix we show that $E_{\infty}$ is of order $N^{-1}$ for large $N$. We define $E_{\infty}$ as the lowest singleparticle energy when the defect potential at $r^{\prime \prime}$ is infinite. As discussed in Appendix A, the eigenvalue condition, in this case, may be written

$$
\sum_{n} \frac{\left|\psi_{n}^{0}\left(r^{\prime \prime}\right)\right|^{2}}{E_{\infty}-E_{n}^{0}}=0, \quad 0 \leq E_{\infty} \leq E_{1}^{0},
$$

where $E_{1}^{0}$ is the energy of the next-to-lowest energy eigenvalue of the perfect crystal. It is clear from Fig. 1 that a solution to Eq. (B1) does exist and is unique. We now set $E_{\infty}=\epsilon / N$, and note that the lowest-energy eigenvalue and eigenvector of the perfect crystal are, respectively, $E_{0}^{0}=0$ and $\psi_{0}^{0}(r)$ $=N^{-1 / 2}$, whence we may write

$$
\frac{1}{\epsilon}=\sum_{n} \frac{\left|\psi_{n}^{0}\left(r^{\prime \prime}\right)\right|^{2}}{E_{n}^{0}-\epsilon / N}
$$

where the prime indicates that the lowest-energy term which gives rise to the left-hand side of Eq. (B2) is to be omitted from the summation. Since $E_{1}^{0}-\epsilon / N$ must be positive, we have the inequality

$$
(1-\gamma \epsilon)^{-1} g_{0}\left(r^{\prime \prime}\right) \geqslant \epsilon^{-1} \geqslant g_{0}\left(r^{\prime \prime}\right),
$$

where

$$
g_{0}\left(r^{\prime \prime}\right)=\sum_{n} \cdot \frac{\left|\psi_{n}^{0}\left(r^{\prime \prime}\right)\right|^{2}}{E_{n}^{0}}
$$

and

$$
\gamma=\left(N E_{1}^{0}\right)^{-1} .
$$

We may write Eq. (B3) as

$$
(1-\gamma \epsilon) g_{0}\left(r^{\prime \prime}\right)^{-1} \leq \epsilon \leq g_{0}\left(r^{\prime \prime}\right)^{-1} .
$$

For one- and two-dimensional systems, $g_{0}(r)$ diverges in the limit $N \rightarrow \infty$. We will adopt the simple, although perhaps over-restrictive, definition of a three-dimensional system as one for which $N_{x} / N_{y}$ and $N_{y} / N_{x}$ remain of order unity as $N \rightarrow \infty$. With this definition it is straightforward to establish bounds for $g_{0}(r)$ for a three-dimensional system of the form

$$
0<A \leq g_{0}(r) \leq B<\infty,
$$

where $A$ and $B$ are constants independent of $N$ and $r$ for sufficiently large $N$. Then since $E_{1}^{0} \sim N^{-2 / 3}$, Eqs. (B4b), (B5), and (B6) imply that for sufficiently large $N$,

$$
\left(1-A^{\prime} / N^{1 / 3}\right) \leq \epsilon g_{0}\left(r^{\prime \prime}\right) \leq 1,
$$

where $A^{\prime}$ is a constant, independent of $N$ and $r$. From Eq. (B7) we conclude that

$$
N E_{\infty} \sim g_{0}\left(r^{\prime \prime}\right)^{-1}, N \rightarrow \infty .
$$

*Work supported in part by the National Science Foundation. 'John Simon Guggenheim Fellow, 1972-1973. Permanent address: Department of Physics, University of Pennsylvania, Philadelphia, Pa. 19104.

${ }^{1}$ P. Soven, Phys. Rev. 156, 809 (1967), D. W. Taylor, Phys. Rev. 156, 1017 (1967).

${ }^{2}$ E. C. Svensson, W. J. L. Buyers, T. M. Holden, R. A. Cowley, and R. W. H. Stephenson, AIP Conf. Proc. 5, 1315 (1972).
${ }^{3}$ I. M. Lifshitz, Adv. Phys. 13, 483 (1964).

${ }^{4}$ S. K. Ma, report of work prior to publication (1973). (Note added in proof. This work has been retracted.)

${ }^{5}$ K. G. Wilson, Phys. Rev. B 4, 3174 (1971).

${ }^{6}$ T. P. Eggarter, Phys. Rev. B 5, 3863 (1972).

${ }^{7}$ R. Courant and D. Hilbert, Methods of Mathematical Physics (Interscience, New York 1953), Vol. I, p. 133.

${ }^{8}$ R. G. Newton, Scattering Theory of Waves and Particles, (McGraw-Hill, New York, 1966). 